\title{
Principal Components Analysis of a JWST NIRSpec Detector Subsystem
}

\author{
Richard G. Arendt ${ }^{b}$, D.J. Fixsen ${ }^{c}$, Matthew A. Greenhouse ${ }^{a}$, Matthew Lander ${ }^{f}$, Don Lindler ${ }^{d}$, \\ Markus Loose ${ }^{e}$, S. H. Moseley ${ }^{a}$, D. Brent Mott ${ }^{g}$, Bernard J. Rauscher ${ }^{a}$, Yiting Wen ${ }^{g}$, Donna \\ V. Wilson ${ }^{f}$, and Christos Xenophontos ${ }^{f}$ \\ ${ }^{a}$ Code 665, NASA Goddard Space Flight Center, Greenbelt, MD \\ ${ }^{b}$ CRESST/UMBC/GSFC, Greenbelt, MD \\ ${ }^{c} \mathrm{CRESST} / \mathrm{UMd} / \mathrm{GSFC}$, Greenbelt, MD \\ ${ }^{d}$ Sigma Space Corporation/GSFC, Greenbelt, MD \\ ${ }^{e}$ Markury Scientific, Inc., 518 Oakhampton Street, Thousand Oaks, CA \\ ${ }^{f}$ Code 582, Goddard Space Flight Center, Greenbelt, MD \\ ${ }^{f}$ Code 553, Goddard Space Flight Center, Greenbelt, MD
}

\begin{abstract}
We present principal component analysis (PCA) of a flight-representative James Webb Space Telescope Near Infrared Spectrograph (NIRSpec) Detector Subsystem. Although our results are specific to NIRSpec and it's $\mathrm{T} \sim 40 \mathrm{~K}$ SIDECAR ASICs and $5 \mu \mathrm{m}$ cutoff H2RG detector arrays, the underlying technical approach is more general. We describe how we measured the system's response to small environmental perturbations by modulating a set of bias voltages and temperature. We used this information to compute the system's principal noise components. Together with information from the astronomical scene, we show how the zeroth principal component can be used to calibrate out the effects of small thermal and electrical instabilities to produce cosmetically cleaner images with significantly less correlated noise. Alternatively, if one were designing a new instrument, one could use a similar PCA approach to inform a set of environmental requirements (temperature stability, electrical stability, etc.) that enabled the planned instrument to meet performance requirements.
\end{abstract}

Keywords: H2RG, cryogenic, SIDECAR, noise

\section{INTRODUCTION}

The James Webb Space Telescope (JWST) Near Infrared Spectrograph (NIRSpec) will be detector noise limited for most observations. To improve NIRSpec's margin for meeting challenging JWST sensitivity requirements, we began studying Improved Reference Sampling and Subtraction (IRS ${ }^{2}$ pronounced "IRS square") in 2010. ${ }^{1-3}$ As described in Sec. 2, IRS ${ }^{2}$ uses a new readout pattern and the measured correlation properties of the hardware to reduce the correlated noise to below what is achievable using "traditional" H2RG readout. However, even using IRS ${ }^{2}$, there was still significant correlated noise in the system.

For the most part, the residual correlated noise manifested as what we call the "H2RG picture frame" (Fig. 1). The picture frame, however, was not new. By the mid-2000s, Don Hall and Marcia Rieke had both noticed that the reference pixels in $J W S T$ 's H2RGs responded differently to temperature than the regular pixels. This was documented in a handful of internal JWST presentations. In 2008, Mike Regan of STScI showed that NIRSpec's H2RG detector array can produce a picture frame artifact when the temperature is not stable. ${ }^{4}$ Our work builds on that of Hall, Rieke, and Regan by modulating a set of biases and temperature to more comprehensively characterize the system's response to environmental disturbance and instability. We used these data for a classical principal components analysis (PCA). By least squares fitting and subtracting the 0th PCA component (i.e. making a "PCA zero correction"), we were able to remove most of the picture frame pattern noise.

Further author information: (Send correspondence to B.J.R.)

B.J.R.: E-mail: Bernard.J.Rauscher@nasa.gov, Telephone: +1 3012864871 


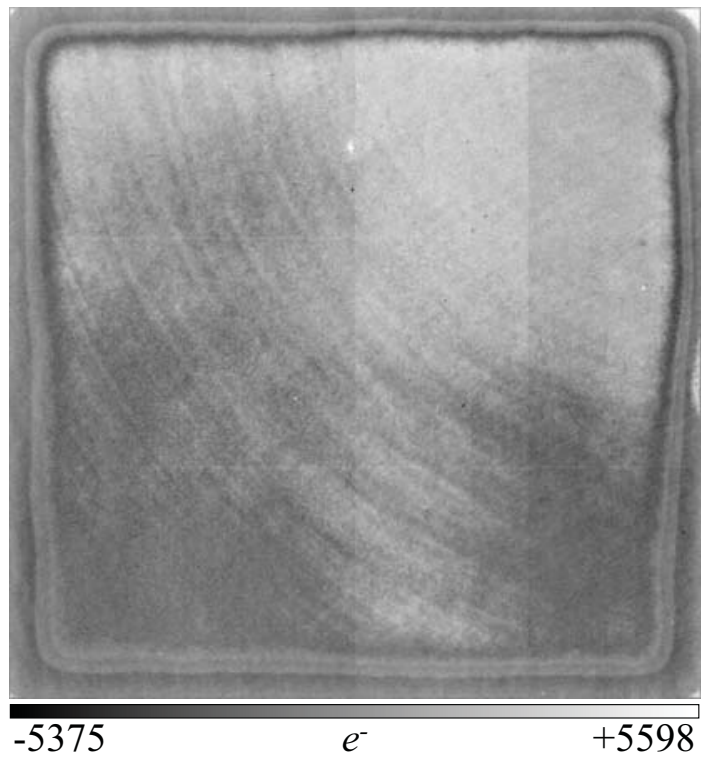

Figure 1. The H2RG "picture frame" is a feature of the readout integrated circuit (ROIC). It can even be seen when physically inspecting a bare ROIC under room light. We speculate that it is caused by small process variations across the ROIC. It is highly correlated from one frame to another sampling up-the-ramp, and so subtracts out reasonably well in correlated double samples (CDS). For NIRSpec, however, even small deviations from perfect correlation are detectable. As described in the text, we use a PCA zero correction to mitigate this. This figure shows one $2048 \times 2048$ pixel frame from a short dark integration. JWST reads its H2RGs using four video outputs which appear as four thick vertical stripes in this figure. These are raw JWST data apart from a very simple reference pixel correction using the average value of reference pixels in rows.

Fig. 2 provides a visual overview of how different kinds of correlated noise appear in dark current images and how they can be removed. Although the cosmetic improvements are important, removing correlated noise also pays dividends in sensitivity. Depending on the astronomical scene, observing strategy, and how sources are photometrically extracted, measurement errors can be reduced by up to $\sim 40 \%$.

The NIRSpec detector subsystem was required to meet challenging environmental requirements may affect the relative importance of the noise contributors compared to what is seen in other H2RG-based systems. To provide context, we provide a brief introduction to the instrument in Sec. 2. Sec. 3 describes the bias modulation experiment and PCA. The ESA-led NIRSpec Instrument Science Team is in the process of studying how IRS ${ }^{2}$ affects NIRSpec sensitivity now.

\section{NIRSPEC}

NIRSpec $^{5}$ is a $0.6-5 \mu \mathrm{m}$ multi-object spectrograph with micro-shutter array (MSA), fixed slit, and integral field unit (IFU) observing modes. It provides spectral resolutions $R=\lambda / \Delta \lambda \in\{100,1000,2700\}$. Our focus in this paper is the DS, which contains two $5 \mu$ m cutoff Teledyne HgCdTe H2RG sensor chip assemblies (SCA). Each SCA is controlled by a Teledyne SIDECAR ASIC that is mounted about $30 \mathrm{~cm}$ away on the cryogenic optical bench. The $\mathrm{T} \approx 40 \mathrm{~K}$ temperature of the SIDECARs and H2RGs is actively controlled to $\leq \pm 1 \mathrm{mK}$ over a timescale of days.

In "traditional" JWST H2RG+SIDECAR readout, pixels are clocked sequentially in the same order in which they appear on the detector array. This closely follows the mode that is described in Teledyne's H2RG manual, and is similar to the readout mode that is used at observatories around the world. For JWST, the detectors are read out differentially and the H2RG's reference output is sent to the ASIC and subtracted with unity gain and no bandwidth limiting from the four video outputs. IRS ${ }^{2}$ differs from traditional readout in two ways: (1) the SCA is read out single-ended and (2) the H2RG's moveable guide window is used to interleave many more 


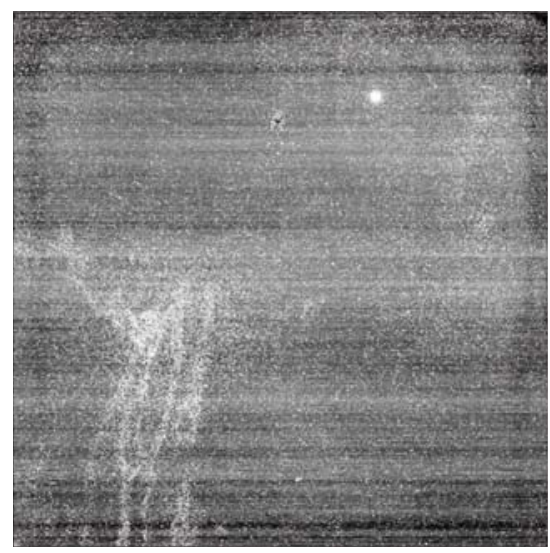

a) Traditional $J W S T$

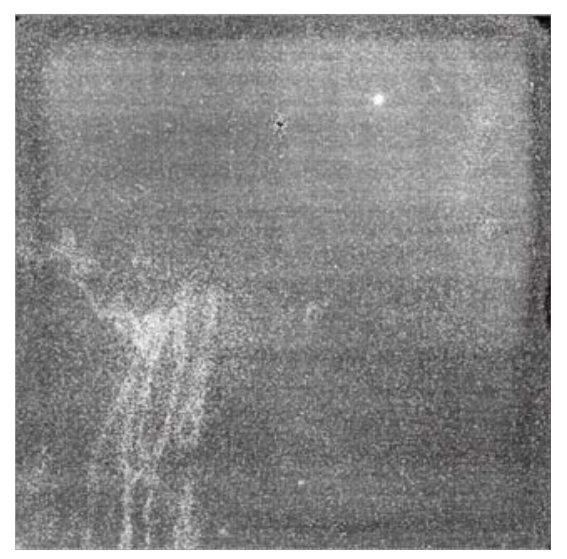

b) $\operatorname{IRS}^{2}$ before 2013

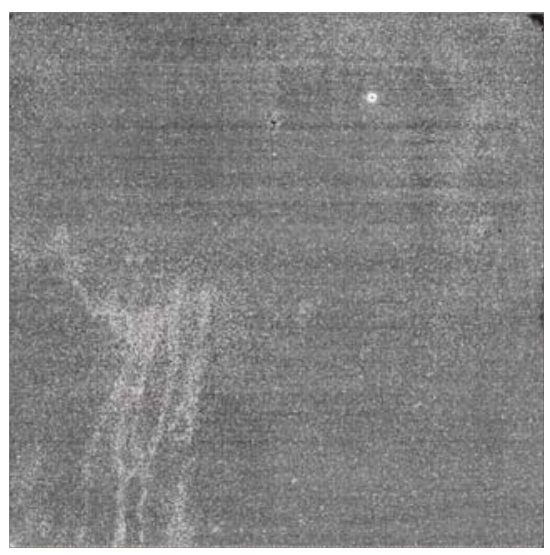

c) $\operatorname{IRS}^{2}$ today

Figure 2. These $\sim 1000$ second dark maps provide a high level view of the noise. All panes are on the same $[-12,+12] e^{-}$ grayscale. When a traditional JWST clocking pattern is used, a), there is obvious correlated noise that appears as faint bars, bands, and a picture frame like pattern. By making more efficient use of the reference output and reference pixels, b), IRS ${ }^{2}$ significantly reduces the bars, bands, and $1 / f$ like noise, but the picture frame remains. When real pixels are used as references for a PCA zero correction in $\operatorname{IRS}^{2}$, c), the picture frame can also be mitigated. These reductions in correlated noise are significant when making photometric measurements. Depending on the astronomical scene and source extraction method, they can reduce the measurement error by up to $\sim 40 \%$.

reference pixels with the regular pixels during readout. ${ }^{1}$ IRS $^{2}$ is still a differential readout mode, however the differencing is done in post-processing using the measured correlation between the reference output and regular outputs.

$J W S T$ places some unusual demands on its near infrared detectors systems. These include running both the H2RG detector arrays and SIDECAR ASICs at T $\sim 40 \mathrm{~K}$. The power dissipation requirements are also challenging. For NIRSpec, each H2RG is required to dissipate $<1 \mathrm{~mW}$ when running with four video outputs at a pixel rate of $100 \mathrm{kHz}$ per output. Each SIDECAR ASIC is only allowed to dissipate $15 \mathrm{~mW}$ when running cryogenically. Although we have not studied how the PCA components change when these requirements are relaxed, we would not be surprised if there were some environmental dependencies.

\section{METHOD}

This section describes the test hardware, bias and temperature modulation experiment (hereafter just "bias modulation"), and PCA. In Sec. 3.4 we provide a short "Quick Start Guide" for the benefit of readers who may wish to quickly try it out on another system before digging into the mathematical details.

\subsection{Pathfinder Detector Subsystem}

The Pathfinder is a complete NIRSpec detector subsystem (Fig. 3) including the focal plane assembly (FPA) with the two $5 \mu \mathrm{m}$ cutoff H2RG SCAs, the interconnect cables that join the FPA to the two cryogenic SIDECAR ASICs, and the FPE that controls the SIDECARs and provides active thermal control of the FPA and SIDECARs.

One of the Pathfinder's two H2RG SCAs, H2RG-S042, has flight representative noise performance; e.g. total noise $=6.6 e^{-}$rms, dark current $=0.003 e^{-} / s$, pixel operability $\approx 98 \%$. For more information on the Pathfinder's performance, please see Tab. 1 of [3]. For the bias modulation test, the Pathfinder was configured to take IRS2-65 integrations, with $r=4$ reference pixels interleaved every $n=16$ normal pixels. In IRS $^{2}$ readout, the clocking pattern "jumps" to the reference pixels in rows every $n$ normal pixels, digitizes $r / 2$ reference pixels, steps to the next reference column, digitizes $r / 2$ reference pixels, and then "jumps" back into the normal pixels to digitize another $n$ normal pixels again. The end result is to interleave many more reference samples into the readout 


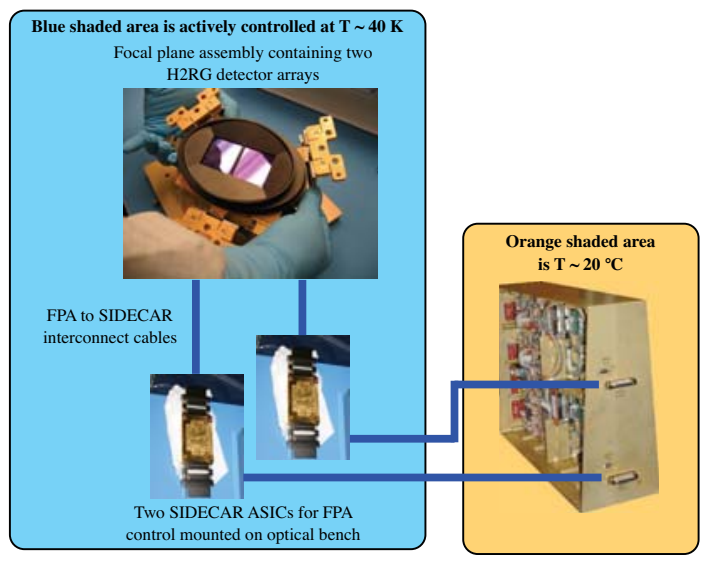

Figure 3. The NIRSpec detector subsystem Pathfinder provides a flight representative testbed for IRS ${ }^{2}$ development. The Pathfinder's FPA contains two $5 \mu \mathrm{m}$ cutoff SCAs. One of the two, H2RG-S042, has flight-like performance apart from a photo-emissive defect that affects one output. The FPA is connected to the two SIDECAR ASICs by an approximately $30 \mathrm{~cm}$ long harness. The FPA, harness, and SIDECARs are all actively controlled at $\mathrm{T} \sim 40 \mathrm{~K}$ by the FPE. The FPE achieves $\pm 1 \mathrm{mK}$ temperature stability on a timescale of weeks. As is described in the text, both the H2RGs and SIDECARs have demanding power dissipation requirements as well.

pattern than would otherwise be possible. We refer the interested readout to an earlier paper ${ }^{3}$ for a diagram and more complete description of the IRS ${ }^{2}$ clocking pattern.

In IRS2-65, there are 65 IRS $^{2}$ sampled up-the-ramp frames. Including overheads, the frame readout time is $14.5889 \mathrm{~s}$ and the integration time is $933.688 \mathrm{~s}$. For the temperature modulation tests, we took long, automated sequences of IRS2-65 integrations. The temperature was varying at all times. For the bias modulation tests, the JWST SIDECAR firmware requires that all bias changes happen before an integration, and the bias must stay constant during that integration. In practice, this means that the bias modulation profiles were quantized with discrete steps before each integration, but with the bias values being held constant during the integrations. We discuss this further in Sec. 3.2.

For the duration of the bias modulation test we bypassed the Pathfinder's thermal control system and used a Lakeshore controller. We did this because the Lakeshore is much easier to reconfigure. The actual $\sim 100 \mathrm{mK}$ temperature changes were so large compared to the $\sim 1 \mathrm{mK}$ thermal control loops that the difference is unimportant.

\subsection{Bias and Temperature Modulation Experiment}

To better understand the picture frame, we measured the DS's response to electrical bias and temperature perturbations and performed PCA (Sec. 3.3). We programmed the DS to modulate one parameter at a time and took long sets of 40 sequential 65-frame up-the-ramp sampled integrations. After some preliminary experiments, we settled on the IRS ${ }^{2}$ parameters $n=16$ and $r=4$ (see Fig. 1 of [3]). This interleaves $r=4$ reference pixels every $n=16$ normal pixels. The elapsed time per exposure is about 16 minutes, resulting in a running time of about 11 hours per bias parameter varied. For the temperature modulation runs, we were manually generating the waveform. The experiment durations were shorter, depending on how much time was available on a given day.

The H2RG bias voltages are described in Teledyne's user manual. Our analysis suggested that VBIASPOWER, VBIASGATE, and DSUB were likely to couple strongly to the SCA output. Because it was straightforward to do, we also varied the output source-follower's DRAIN and VRESET. Due to detector subsystem software constraints, we were not able to continuously vary the bias voltages. Instead, for the biases, we changed the value before taking each 65 frame exposure. We used a Lakeshore controller to vary the temperature, so in this case we were able to generate a continuous profile by manually programming the Lakeshore to execute ramps up and 


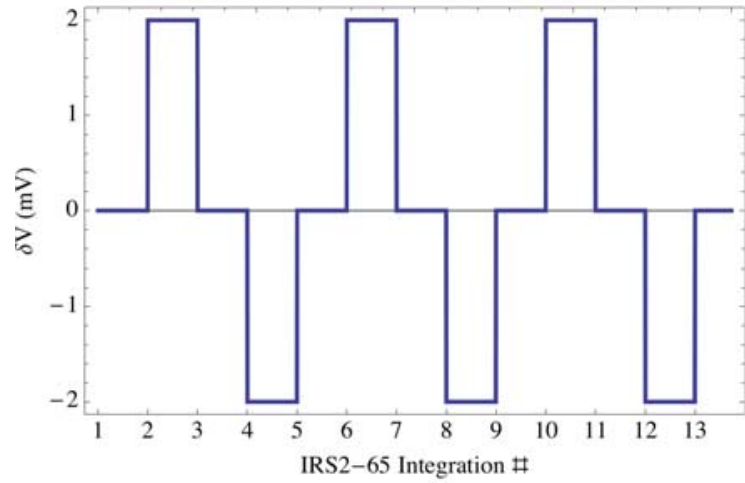

a) Bias modulation

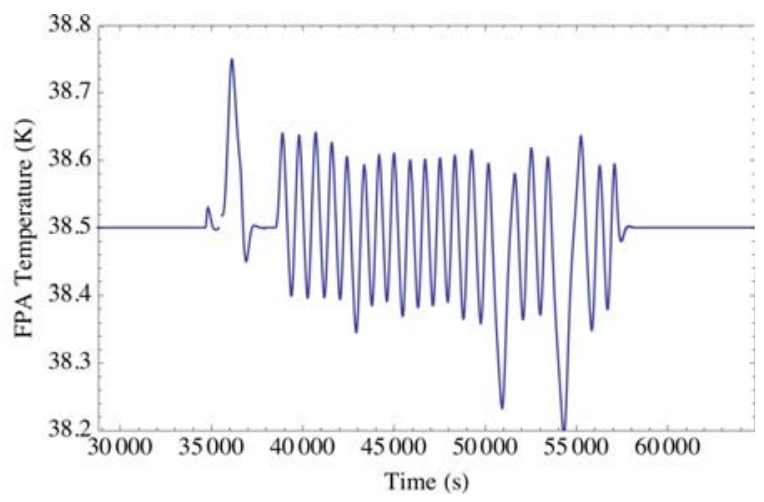

b) Temperature modulation

Figure 4. a) In bias modulation, we programmed the SIDECAR ASIC to change one bias by about $1 \mathrm{mV}$ before each IRS2-65 integration. The resulting profiles were quantized. b) In temperature modulation, we used a handheld timer and manually programmed the Lakeshore temperature controller to ramp up/down with an amplitude of about $100 \mathrm{mK}$. The resulting profiles were not perfect, but they did not have to be. The only needed to provide the information necessary compute the differential responses of Eq. 2

down. Fig. 4 shows a few representative modulation profiles. In total, we modulated five bias voltages plus temperature.

\subsection{Principal Components Analysis}

Throughout this article we use the Einstein summation convention unless stated otherwise. Repeated indices indicate a sum over all array elements. Greek indices run over the six parameters that we modulate and roman indices run over all $2040^{2}$ normal pixels in the image area. We use the Kronecker delta as the metric for raising and lowering indices.

Apart from temperature, which we had to modulate manually, all of the other tests were automated. We spent about one day collecting data for each parameter, and the full experiment required about one week of lab test time. The experiment produced six data sets, one for each parameter varied,

$$
b_{\alpha}=(\text { VBIASPOWER, VBIASGATE, VRESET, DSUB, DRAIN, Temperature }) .
$$

Let $D^{i}$ be a vector representing a $2040 \times 2040$ pixel frame of IRS ${ }^{2}$ reference corrected data. Because the reference pixels have been trimmed off at this point, only the $2040^{2}$ normal pixels remain. See [3] for a description of IRS ${ }^{2}$ reference correction. For dark images, these input data are essentially what a calibration pipeline would deliver to an astronomer for image analysis.

We used these data to compute the system's linear differential response with respect to each stimulus, or in other words the Jacobian matrix,

$$
J_{\alpha}^{i}=\left(\frac{\partial}{\partial b_{1}}, \frac{\partial}{\partial b_{2}}, \frac{\partial}{\partial b_{3}}, \frac{\partial}{\partial b_{4}}, \frac{\partial}{\partial b_{5}}, \frac{\partial}{\partial b_{6}}\right) D^{i},
$$

Fig. 5 shows the six partial derivatives. These are the six columns of $J_{\alpha}^{i}$. Although the Jacobian provides a complete picture of the system's first order linear response to these small perturbations, it does not provide any information on which aspects were more or less important. Moreover, it contains many correlations. By rotating from the six dimensional coordinate system of the Jacobian to an orthonormal representation, we can obtain a ranked set of principal components.

Using Eq. 2, to compute the $6 \times 6$ element autocorrelation matrix,

$$
v_{\alpha \beta}=J_{\alpha}^{i} \delta_{\mathrm{ij}} J_{\beta}^{j} .
$$

To complete the PCA, we computed the eigensystem of $v_{\alpha \beta}$. PCA zero is the eigenvector that is associated with the largest eigenvalue. Fig. 6 shows the six computed principal components. These provide an orthonormal basis for expressing the system's first order linear response to these six stimuli. 


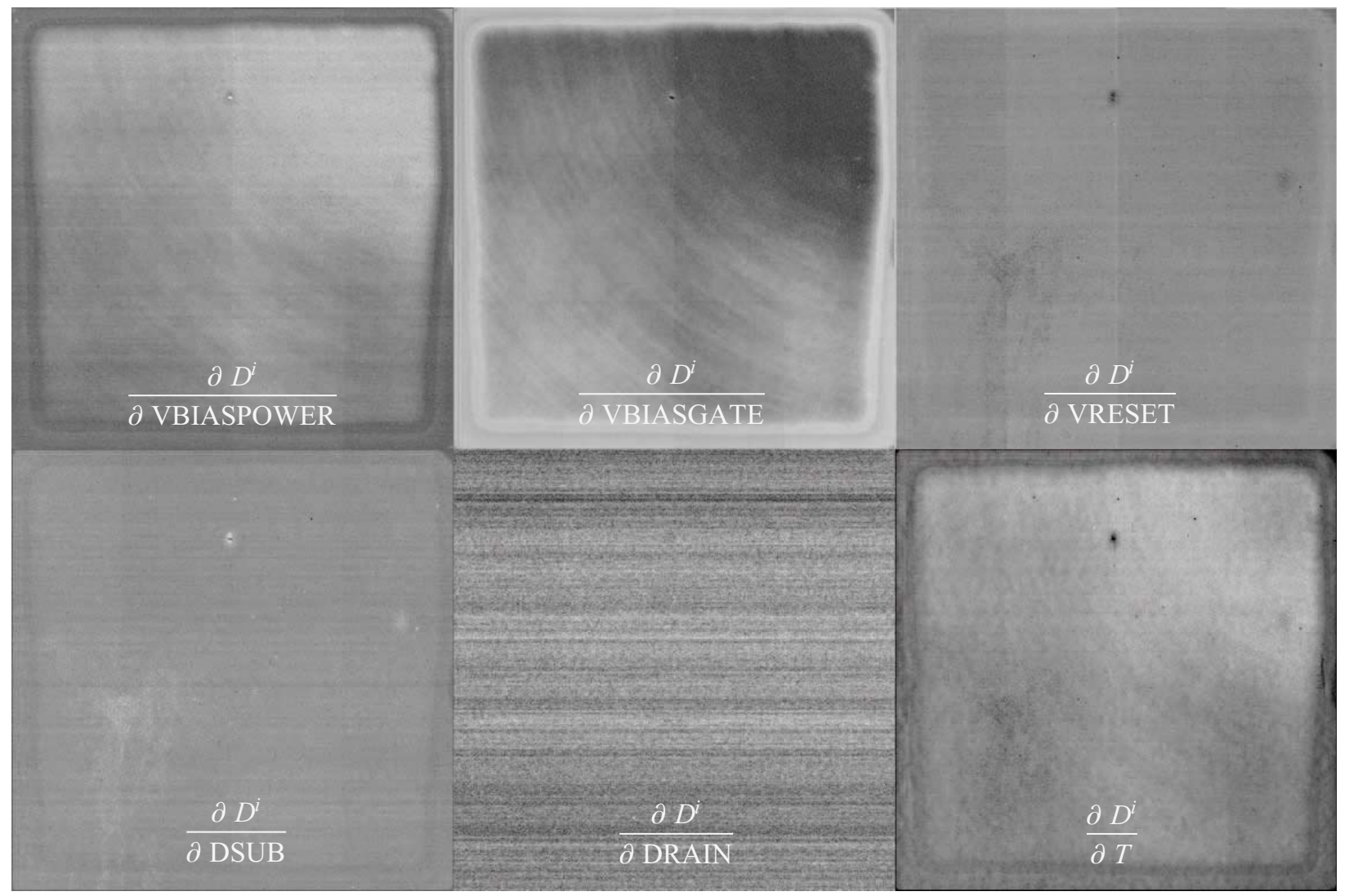

Figure 5. For the PCA, we modulated five bias voltages and temperature. We used these data to compute the differential linear response with respect to each parameter. These inputs to the PCA show that VBIASPOWER, VBIASGATE, and temperature can all drive the picture frame.

\subsection{Quick Start Guide}

This section is provided for the benefit of readers who may wish to try PCA out for themselves before digging further into the details. The basic idea of bias modulation is to comprehensively measure the detector system's response to small environmental perturbations and to use this information to find a representation that is free of correlations. In our work, we modulated temperature and a set of five biases. More biases would provide more information, but at minimum we recommend modulating: (1) temperature, (2) VBIASGATE, VBIASPOWER, and DSUB. The steps are as follows.

1. Take dark data while modulating one parameter at a time.

2. Compute the partial derivative of the data output values with respect to each parameter that was. This should produce a set of images like Fig. 5

3. Reformat each of the partial derivative images into a vector and put all of the vectors into a matrix. Each row in the matrix should contain the partial derivative of the output with respect to one parameter. In our case, we modulated six parameters and our matrix had six rows and $2040^{2}$ columns.

4. Multiply this matrix by its own transpose to yield the autocorrelation matrix.

5. The eigenvectors of the autocorrelation matrix are the principal components. 


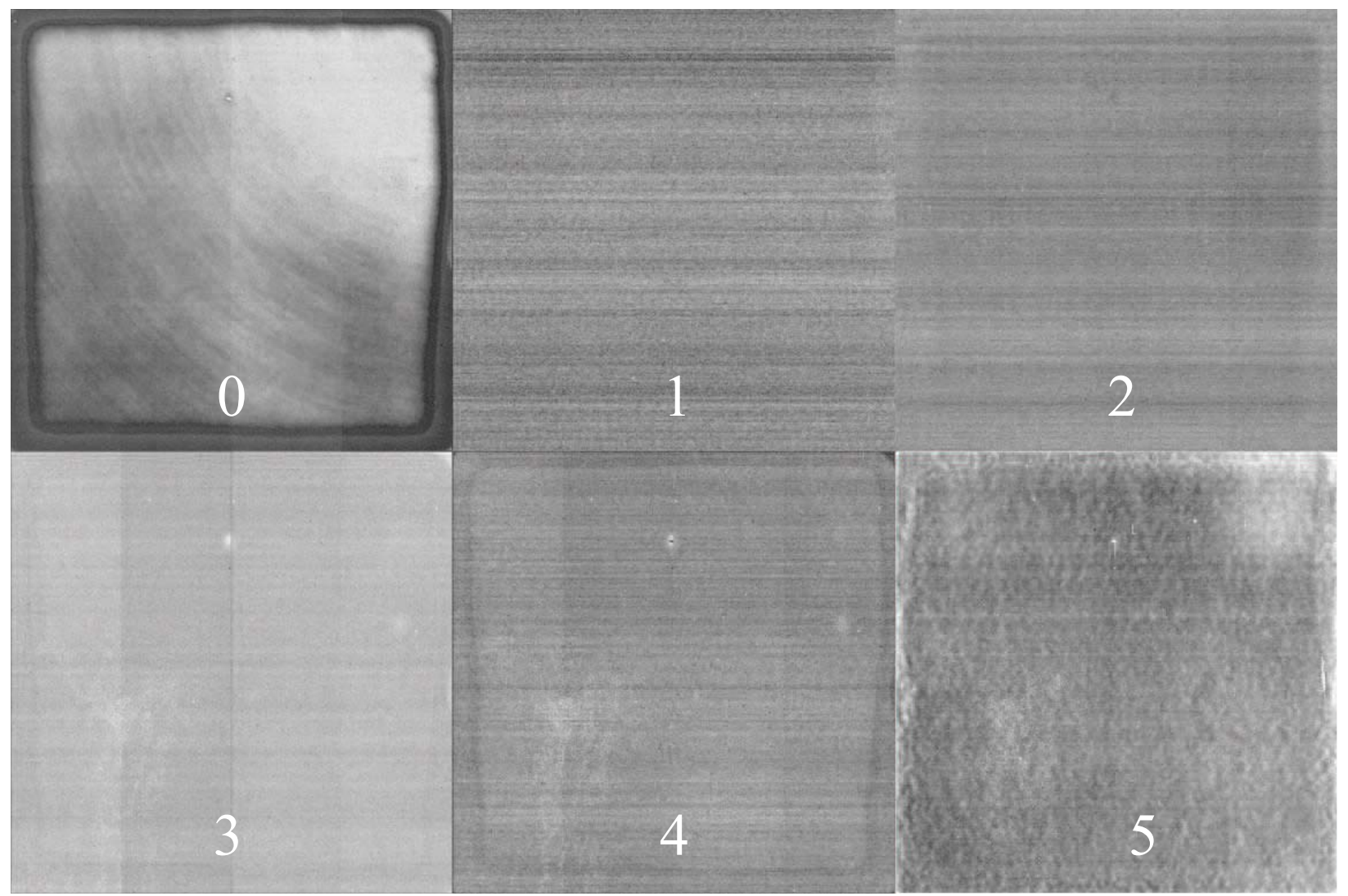

Figure 6. The PCA components are the eigenvectors of the autocorrelation matrix, $v_{\alpha \beta}$ (Eq. 3). They are: (0) the picture frame (the only strong picture frame), (1) $1 / f$ which is almost exactly $\partial D^{i} / \partial \mathrm{DRAIN},(2)$ a different $1 / f$ that come from a mix of $\partial D^{i} / \partial$ VBIASPOWER and $\partial D^{i} / \partial$ VBIASGATE, $(3) 1 / f+\partial D^{i} / \partial$ VRESET artifacts $(+$ bad pixels $)(4) 1 / f+$ $\partial D^{i} / \partial \mathrm{DSUB}$ artifacts $(+$ bad pixels $)+$ some residual picture frames. (5) the mottled component of $\partial D^{i} / \partial T$ (with the picture frame removed).

\section{REMOVING THE PICTURE FRAME}

Once we realized that the picture frame was the 0th PCA component in IRS ${ }^{2}$ processed images, we set about removing it using blanked off real pixels in the scene as references. The simpleset approach, directly fitting PCA zero to a set of blanked off pixels, did not work very well. The reason was evident when we blinked though a set of $40 \sim 1000 s$ IRS $^{2}$ processed dark maps. The picture frame is not constant, its amplitude is a function of time because all of the parameters that drive it ( $T$, VBIASPOWER, and VBIASGATE, etc.) are slowly varying functions of time.

We therefore fitted and subtracted PCA zero, $0^{i}$, modulated by time,

$$
D^{\prime i}=D^{i}-\gamma(t) 0^{i},
$$

where $\gamma$ is a slowly varying functiont that we approximate with a few low-order sine and cosine terms,

$$
\gamma(t)=\sum_{k=1}^{n} \gamma_{k} \exp 2 \pi k\left(\frac{t}{t_{f}}\right) i .
$$

In Eq. 5 the summation convention does not apply. $k$ is just a subscript and $t_{f}$ is the frame readout time.

Panel C of Fig. 2 shows the result of applying Eq. 4 with $n=12$ to the NIRSpec Pathfinder data. If "total noise", which is equal to the square root of the variance is used as a figure of merit, the total noise is about 
$(6.1,5.3,4.9) e^{-}$rms in panels $\mathrm{A}, \mathrm{B}$, and $\mathrm{C}$ respectively. However, total noise considers only the diagonal elements of the covariance matrix. For more realistic observing scenarios which use sky subtraction to infer source brightnesses, we believe that the advantages are greater still. As was mentioned earlier, the NIRSpec Science Team is working to quantify the relative advantage for realistic NIRSpec observing scenarios now.

\section{SUMMARY}

We have presented PCA of a flight representative JWST NIRSpec detector subsystem. The Pathfinder" detector system uses a pair of cryogenic $5 \mu \mathrm{m}$ cutoff H2RG detectors and SIDECAR ASICs, both operated at T $\sim 40 \mathrm{~K}$ and optimized for very low power dissipation. Even with temperature stabilized to $\pm 1 \mathrm{mK}$ on a timescale of days, we show that the system's most important residual principal noise component after IRS ${ }^{2}$ reference correction is the H2RG picture frame. We show that under NIRSpec's operating conditions, the picture frame is pumped by small environmental instabilities, with temperature, VBIASPOWER and VBIASGATE all contributing significantly. Building on this, we show how the picture frame can be removed by using real pixels as references and fitting the time modulated 0th PCA component, "PCA zero".

This is our third SPIE paper on IRS ${ }^{2}$. Looking back, the first paper ${ }^{1}$ presented the underlying theory and papers 2-3 showed how IRS ${ }^{2}$ was able to reduce NIRSpec's read noise by about $14 \%$ compared to the traditional H2RG readout that is described in Teledyne's manual and in wide use at observatories today. ${ }^{2,3}$ This article builds on our earlier work by invoking real pixels as references and using PCA to further reduce the read noise to about $20 \%$ below what would be achieved using traditional readout.

However, these comparisons consider only the diagonal (uncorrelated) terms of the covariance matrix. We believe that when realistic observing scenarios are considered, the off diagonal terms which appear when e.g. sky subtraction is done, will increase the relative advantage of IRS ${ }^{2}$. As of today, the NIRSpec Instrument Science Team is evaluating IRS ${ }^{2}$ in this regard. We are also working with the ESA Instrument Team on how to best incorporate reference correction (including the PCA zero correction that is described here) into NIRSpec data reduction and analysis.

\section{ACKNOWLEDGMENTS}

This work was funded by NASA as part of the James Webb Space Telescope Project.

\section{REFERENCES}

[1] S. H. Moseley, R. G. Arendt, D. J. Fixsen, D. Lindler, M. Loose, and B. J. Rauscher, "Reducing the read noise of H2RG detector arrays: eliminating correlated noise with efficient use of reference signals," Proc SPIE 7742, p. 36, July 2010.

[2] B. J. Rauscher, R. G. Arendt, D. J. Fixen, M. Lander, D. Lindler, M. Loose, S. H. Moseley, D. V. Wilson, and C. Xenophontos, "Reducing the read noise of HAWAII-2RG based detector systems with improved reference sampling and subtraction," Proc SPIE 8155, p. 45, Sept. 2011.

[3] B. J. Rauscher, R. G. Arendt, D. J. Fixsen, M. Lander, D. Lindler, M. Loose, S. H. Moseley, D. V. Wilson, and C. Xenophontos, "Reducing the read noise of HAWAII-2RG detector systems with improved reference sampling and subtraction," Proc SPIE 8453, pp. 84531F-84531F-11, Sept. 2012.

[4] M. W. Regan, L. E. Bergeron, M. Robberto, K. Lindsay, and D. Long, "Determining Detector Temperature using a Self-Calibration Least Squares Process," STScI Technical Report JWST-STScI-001467, SM-12 , pp. 1-11, June 2008.

[5] S. M. Birkmann, T. Böker, P. Jakobsen, G. De Marchi, M. Sirianni, G. Bagnasco, P. L. Jensen, M. B. J. Te Plate, P. Rumler, J.-C. Salvignol, P. Strada, M. Kolm, X. Gnata, K. Honnen, J. Köhler, R. Lemke, M. Maschmann, M. Melf, J.-F. Pittet, P. Ferruit, and B. Dorner, "Status of the NIRSpec instrument," Space Telescopes and Instrumentation 2010: Optical 7731, p. 9, July 2010. 